\title{
Optimization of surface plasmon resonance based biosensor for clinical diagnosis of the Epstein-Barr herpes virus disease
}

\author{
R.V. Khrystosenko \\ V. Lashkaryov Institute of Semiconductor Physics, NAS of Ukraine, \\ 41, prospect Nauky, 03028 Kyiv, Ukraine \\ E-mail: khristosenko@ukr.net
}

\begin{abstract}
The influence of the sensitive element fabrication technology and noise performance of surface plasmon resonance (SPR) immunosensor on sensitivity and stability of operation inherent to the "Plasmon" series instrument has been investigated to improve reliability of diagnosis and treatment of the Epstein-Barr herpes virus disease. To minimize the effect of the temperature change induced noise, compensation (introduction of the reference channel) and stabilization (active thermal control) methods were applied, allowing to substantially enhance resolution of the used SPR sensor system.
\end{abstract}

Keywords: Epstein-Barr virus (EBV), antibodies, surface plasmon resonance (SPR), sensitive element, noise effect.

Manuscript received 10.11.15; revised version received 21.01.16; accepted for publication 16.03.16; published online 08.04.16.

\section{Introduction}

For several recent decades, there observed is a growing interest to the miniature, cheap and sensitive optical transducers for direct detection of molecular interactions in a real time scale without molecular labeling, and application of these transducers in the fields such as environment and industrial waste monitoring, production quality assessment, discovery of new drugs and clinical diagnosis [1]. In these sensors, one type of interacting molecules is immobilized on the transducer surface, forming a sensitive element, and the binding of a complementary molecule to it is monitored measuring the changes in optical density at the sensor surface. The surface plasmon resonance (SPR) method is one of the most advanced and well-developed optical sensing techniques and is widely applied for detection of chemical and biological substances [2]. However, up to this date the
SPR-based instruments suffer from insufficient performance, sensitivity, and high production cost [3].

Surface plasmons (SP) are the normal modes of charge density existing at the interface between dielectric and metal [4]. Conditions for the resonant coupling of SP and electromagnetic field of exciting light are extremely sensitive to the changes in optical properties of dielectric medium near the metal surface. These conditions are characterized by the surface plasmon resonance phenomenon and can be registered using various configurations for surface electromagnetic wave excitation (prism or grid) and various methods for measuring the metal/dielectric interface reflectance (scanning the angle of incidence at the fixed wavelength, or wavelength scanning for the fixed angle of incidence, or combining both of them).

Since the spreading of viral infections grows inexorably, adversely affecting human health, one of the 
important fields of SPR method application is rapid and accurate detection of viral agents and antibodies in blood serum [5-7].

The Epstein-Barr virus (EBV, $4^{\text {th }}$ type human herpevirus) is one of the most widespread and dangerous human viruses, named after the English virologist prof. Michael Anthony Epstein and his graduate student Yvonne Barr, who described the virus in 1964 [8]. A clinical form of initial EBV infection is the infectious mononucleosis that affects lymphatic and nervous systems [9]. The EBV facilitates development of chronic fatigue, autoimmune disorders and cancer [10]. In majority of cases, the disease is asymptomatic, however like to the other herpevirae EBV activates during immunodeficiencies of various origins (HIV infection, cancer, hard radiation or stress) and can by itself become a cause for immunosuppression.

The purpose of this work was to investigate the influence of sensitive elements preparation technology and noise characteristics of the SPR immunosensor on sensitivity and stability of "Plasmon" series instrument operation for reliable detection of EBV antibodies in the human blood serum.

\section{Methods and materials}

Methods. In clinical practice, molecular biology methods such as polymerase chain reaction (PCR), immunofluorescence, and enzyme-linked immunesorbent assay (ELISA) are used for EBV infection diagnosis. The latter method is the most suitable for large-scale analysis, however, it is expensive and timeconsuming [11]. All these methods are based on a specific antigen-antibody interaction. Antibodies are soluble proteins (immunoglobulins) produced by B-lymphocytes in response to the presence of external or intrasystem antigens. They selectively bind to the antigens with formation of an inert antigen-antibody complex, thus blocking the antigen interaction with the other host molecules. Presence of the specific antibodies in circulating system can serve as a marker of various diseases, such as microbial or viral infection, allergy, autoimmune disorder or damaged tissue.

In this work, the surface plasmon resonance spectroscopy method with prismatic excitation in the thin gold film in Kretschmann configuration with mechanical angular scanning was used for detection of EBV antibodies in the human blood serum. The method allows to investigate kinetics of molecular interaction without labeling by means of the measurement of SPR resonant angle in real time with high sensitivity.

The gold films were deposited on glass substrates without heating by thermal evaporation in vacuum (the VUP-4 setup with the residual pressure close to $4 \cdot 10^{-4} \mathrm{~Pa}$, deposition rate of $40 \ldots 50 \AA / \mathrm{s}$ ), with the thin chromium layer ( $\sim \mathrm{nm})$ used to improve adhesion.

Sensitive materials. The Epstein-Barr virus (antigen) was accumulated and extracted from the B95-8 cell culture using the Walls and Crawford method [12] at the D. Zabolotny Institute of Microbiology and Virology of NAS of Ukraine. The B95-8 cell culture consists of the B-lymphocytes from peripheral blood of marmoset monkeys transformed with EBV and continuously producing the virus. Lysates of the lymphoblast B95-8 cells were used as the antigen, containing the full spectrum of EBV proteins, namely EBNA, EA and VCA. This way, a specific antigen for the detection of Epstein-Barr virus antibodies (IgG and $\operatorname{IgM}$ ) was obtained, having high specificity and sensitivity properties. To evaluate the sensitivity and specificity of the obtained antigen, the reproducibility of positive and negative analysis results for the selected panels of respectively positive and negative sera was compared using our test system and several foreign test systems utilizing PCR (“АмплиСенс-100-R", Russia) and ELISA ("Platelia EBV EBNA IgG" from "Sanofi diagnostics Pasteur", France; "SIA ${ }^{\mathrm{TM}}$ Epstein-Barr EBNA IgG" from "Sigma diagnostics", USA).

Analytes. The blood sera from the infected donors were supplied by “ДНК-лаборатория”, Ltd. (Kiev), and sera taken from healthy donors were supplied by Kiev city blood transfusion station. All sera were tested for the presence of EBV antibodies by means of PCR and ELISA reference methods.

\section{Results and discussion}

In the case when SPR method is used for detection of biomolecules in liquid medium, sensitivity can be enhanced by improving the sensitive element preparation technology and by minimizing the effect of the noise.

Preparation of the SPR sensitive element. Enhancement of the method sensitivity is based, first of all, on the specific interaction of EBV antibodies, circulating in the blood serum, with the antigen (EBV itself) immobilized on the gold film surface. The use of immune molecular reaction of the antigen-antibody interaction as the basis for biosensor design allows to obtain uniquely specific responses and to perform analyses of the complex biological samples such as urine, saliva, blood or serum [13].

However, adsorption of biomolecules onto the raw gold surface can lead to undesirable optical and structural effects of metallic surface that distort the SPR instrument response. On the other hand, in order to obtain reliable analysis results the EBV protein molecule has to be immobilized on the gold surface while preserving its natural state and accessible spatial orientation, via an intermediate transition layer. The maximum sensitivity of transducer can be achieved, if the transition layer thickness is substantially smaller than the distance of surface plasmon wave penetration to the external environment $(100 \ldots 200 \mathrm{~nm}$ for visible light).

To form a protective layer on the sensitive element, self-organized monolayers (SOM) with controlled 
composition, structure and thickness can be used. The most widespread are layers based on functionalized mercaptans, particularly aliphatic thiols $\left(\mathrm{HS}-\left(\mathrm{CH}_{2}\right)_{\mathrm{n}}-\mathrm{X}\right)$ due to simplicity of preparation, stability and functional diversity. The primary idea of application of these linear molecules consists in usage of gold affinity to sulfur atoms. Organization of the modifying layer on the noble metal surface happens as a result of prolonged spontaneous chemisorption from solution, predominantly in ethanol, with the concentration $(0.1 \ldots 1.0) \cdot 10^{-3} \mathrm{M}$ and includes two stages. Initially the HS group of thiol interacts with the gold atoms on the surface, forming the gold thiolate. After this, microstructural reorganization of the bound monolayer takes place, controlled by hydrophobic interaction of the aliphatic chains and accompanied by reconstruction of the noble metal surface. This way, a densely packed molecular coating is formed, providing a significant barrier for electron and ion transfer, and possessing high kinetic and thermodynamic stability. The terminal functional groups determine physical and chemical properties of the modified metal surface, and hydrocarbon chains provide spatial shielding up to tens of angstroms (characteristic length of a $\mathrm{C}-\mathrm{H}$ bond is $\sim 1.1 \AA$ ), which does not significantly affects the transducer sensitivity.

Protective properties of the dodecanthiol film $\left(\mathrm{HS}\left(\mathrm{CH}_{2}\right)_{11} \mathrm{CH}_{3}\right)$ are illustrated in Fig. 1. It can be seen that the baseline of the SPR instrument with the applied modifier coating is more stable, monotonous and shows the trend of slight increase with time $(\sim 32$ angular seconds per hour), which may indicate the presence of small quantity of adsorbate substances in water. For the unprotected gold film, the shift of SPR minimum position toward the lower angles can be observed ( $\sim 88$ angular seconds per hour), likely related to the water pervasion through the pores and microcrystal boundaries in the gold film.

Based on the above, the following method was developed for preparation of the sensitive element (chip) for detection of the Epstein-Barr virus antibodies in human blood serum [14]. Immobilization of EBV proteins was performed within the protective-orienting coating (three-dimensional structure of a polysaccharide hydrogel - Dextran 17000 from Sigma) deposited onto the gold film surface on glass substrate from the solution in $0.05 \%$ citrate buffer at $\mathrm{pH} 5.0-5.2$ with the concentration $2 \mathrm{mg} / \mathrm{ml}$ for 5 hours at the temperature $20 \ldots 25^{\circ} \mathrm{C}$. After washing in the citrate buffer, solution of the antigen in citrate buffer was left on the surface at the temperature of $4 \ldots 8{ }^{\circ} \mathrm{C}$ for $18 \ldots 24$ hours. To prevent nonspecific interaction, after immobilization of the EBV proteins, remaining free sites were blocked using the $1 \%$ solution of bovine serum albumin (BSA) in citrate buffer for 1 hour at the temperature $20 \ldots .25^{\circ} \mathrm{C}$. After that, the chips were rinsed with citrate buffer, dried with the clean air flow and stored at $4 \ldots 8{ }^{\circ} \mathrm{C}$ in the small sterile sealed containers.

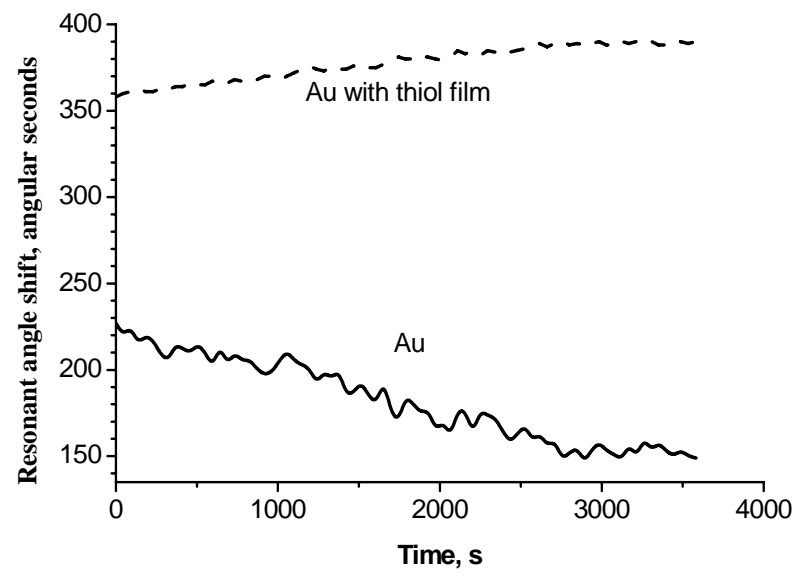

Fig. 1. Temporal drift of the "Plasmon" series instrument baseline in water for the sensitive elements with the raw gold surface and surface modified with dodecanthiol.

Influence of the noise effects. The total noise of a SPR based sensor, determining the lower limit for the measurable changes in the refraction index [15], includes: the photodetector noise; light source fluctuations, largely conditioned by the electronic noise and the quality of controlling circuitry; thermal noise, caused by temperature fluctuations; mechanical fluctuations noise that is a result of internal and external forces applied to the sensor chip and the optical system; the interphase fluctuations noise caused by random sorption-desorption processes at the interface, including the effects from the microscopic air bubbles at the gold film surface, flow/pressure fluctuations caused by the sample supplying pump operation, and the electromagnetic interference from the external sources, affecting the instrument electronics.

The most significant are the effects of temperature fluctuations of different origins [16]. First of all, thermal changes in the gold layer can significantly influence on SP generation. As the temperature increases, SP oscillations substantially weaken due to the increased electron-phonon scattering in metal. It leads to widening the SPR curve and decreasing the instrument sensitivity. The temperature change influences operation of important components of the sensory system (light source output signal, detector response and sensor geometry). However, the parameter most sensitive to the change in ambient temperature is the effective refractive index of the sample. For instance, the water refractive index changes by $10^{-5}$, when the temperature changes by $0.1{ }^{\circ} \mathrm{C}$, which is close to the SPR instruments detection limit [17].

To eliminate the noise caused by temperature fluctuations, stabilization (active temperature control) and compensation (reference channel introduction) principles are used. 
For the implementation of temperature stabilization principle, the thermal stabilization box completely enclosing the operating "Plasmon" series instrument was used (Fig. 2). The box controls the temperature within 2 to $40{ }^{\circ} \mathrm{C}$ range with the accuracy close to $0.1^{\circ} \mathrm{C}$, and uses the developed at V. Lashkaryov Institute of Semiconductor Physics of NAS of Ukraine thermostat with optimized parameters of the proportional differentialintegral controller that modulates the input power to the Peltier-effect based thermoelectric module.

Fig. 3 demonstrates that temperature stabilization of the entire instrument facilitates minimization of the noise level and substantially increases resolution of the used SPR sensor system. However, this approach results in a bulky setup, degrades convenience for performing the experiment, and thus is used, as a rule, only for detection of small molecules at low concentrations.

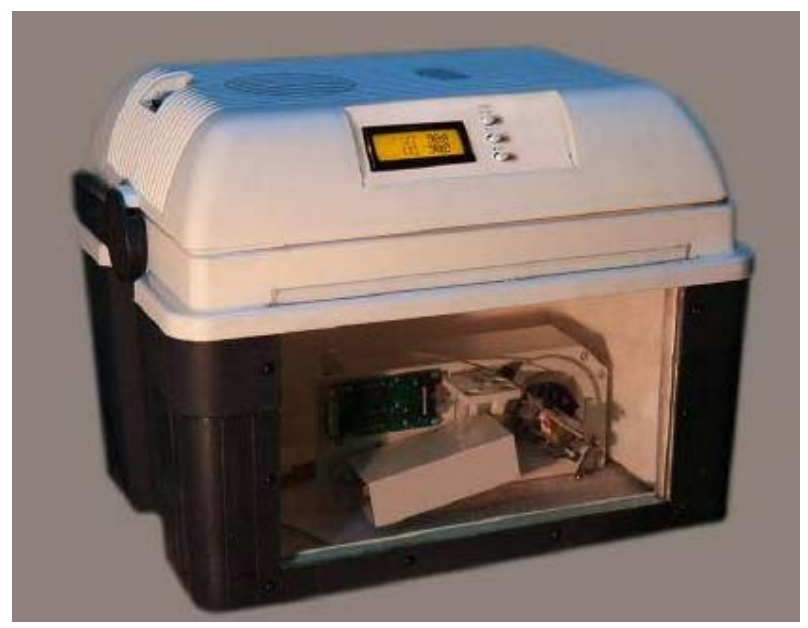

Fig. 2. Exterior view of the thermal stabilization chamber with the "Plasmon" series instrument during operation.

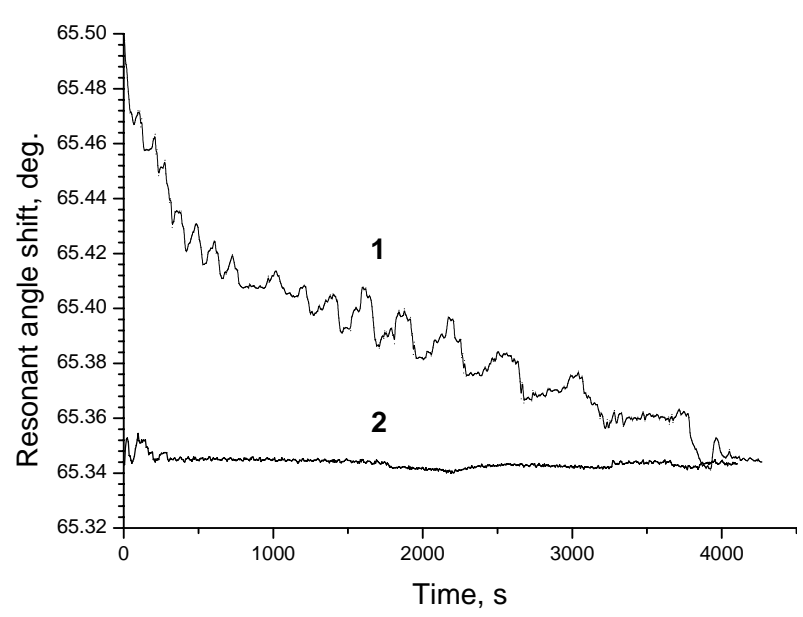

Fig. 3. Long-term drift of the SPR signal in water, obtained with the "Plasmon" series instrument using the described sensor chip: 1 - operating in a non-isolated environment; 2 enclosed within the thermal stabilization box.
For clinical detection of EBV antibodies in human blood serum, the beforehand prepared biochips with EBV proteins immobilized on the gold film surface were used with the "Plasmon" series instrument equipped with an additional reference channel, which readings are registered simultaneously with the primary working channel [18]. Using the reference channel, the changes in the instrument response that are not related to adsorption or molecular interaction (e.g., the temperature changes) are registered, which leads to the improvement of accuracy and reliability of measurements.

Optical layout of the dual channel version of the "Plasmon" series instrument is shown in Fig. 4. The instrument uses a single light source, with the beam being split into two with the specially designed beam-splitting prism. The distance between the axes of beams is $7.5 \mathrm{~mm}$. The beam-splitting prism is the main element of the layout, responsible for formation of the two identically polarized light beams, without refraction and with the spatial shift relative to the light source axis. Only under these conditions, identical SPR curves can be obtained in two channels. The detailed description of "Plasmon" series instrument operation can be found in [19].

In the experiment, an optimal dilution ratio of 1:100 was used for the anti-VBE sera in citrate buffer at pH 5.0...5.5. The channel 1 was used directly for measuring the antibodies content in serum, while the channel 2 was used as the reference one to improve accuracy and reliability of these measurements. To prevent nonspecific binding of antibodies to EBV, negative serum from a healthy donor was supplied to the sample cell first. The unbound material was then washed out, and the serum from an EBV infected donor was supplied to the cell. Quantification of the antigen-antibody interaction results was performed by quantitative determination of the resonant angle shift (in angular seconds) in time (seconds). The sensogram of the measurements is shown in Fig. 5.

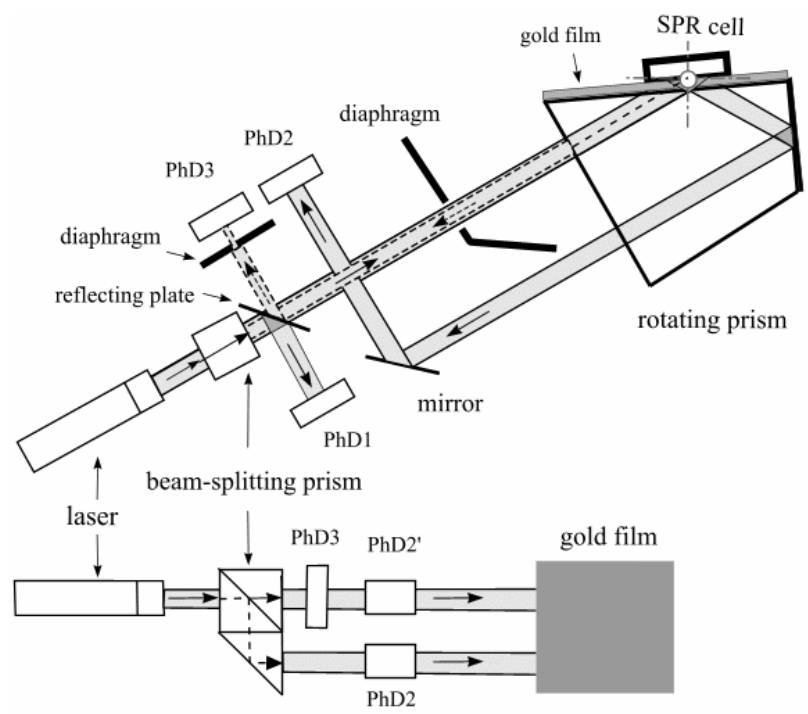

Fig. 4. Optical layout of the dual channel "Plasmon" series instrument. 


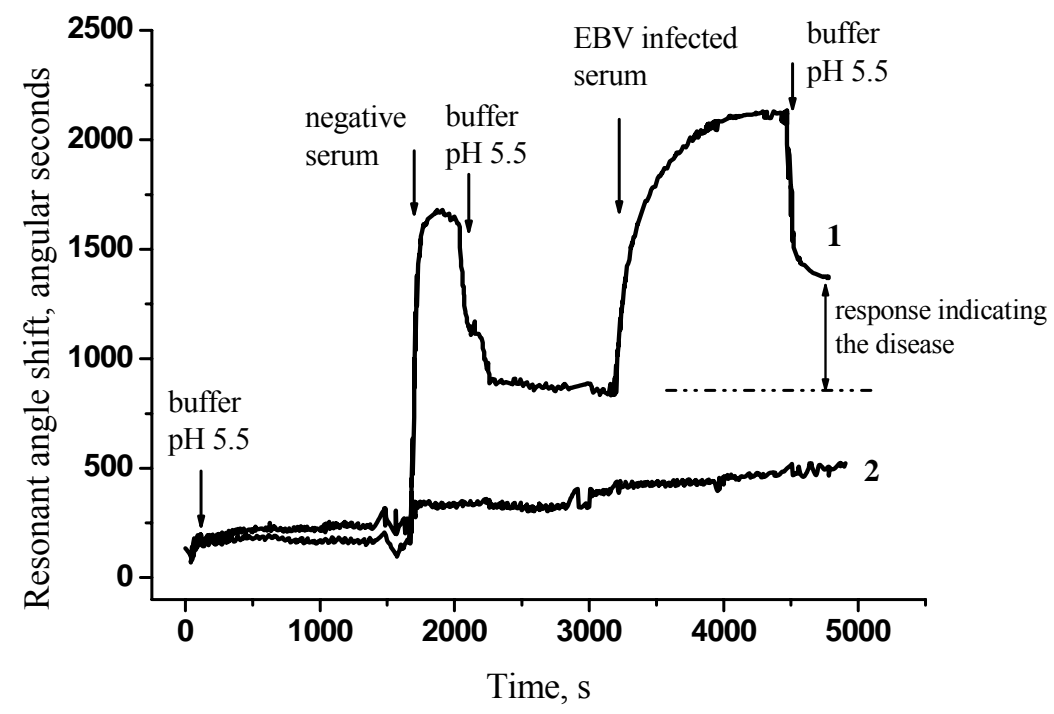

Fig. 5. Sensogram of interaction of the specific antigen (EBV) immobilized on the gold surface of the SPR chip with antibodies in the human blood serum: 1 - operation channel, 2 - reference channel.

The routine analysis is performed as follows. From the average value obtained over 25 negative sera with three measurements for each serum, the threshold value $(\mathrm{ThV})$ is calculated. The serum is considered positive if the resonant angle shift exceeds $\mathrm{ThV}$ by $10 \%$, and negative, if the shift is by $10 \%$ lower than ThV. The obtained data were compared with the ELISA results. Reproducibility of the proposed method outcomes was about $95 \%$.

\section{Conclusion}

Analysis of the influence of SPR sensitive element preparation technology and noise effects of the "Plasmon" series instruments has been performed in order to reliably detect specific antibodies against the Epstein-Barr herpevirus in the human blood serum.

To minimize the influence of temperature fluctuations, the instrument with an additional reference channel was used, the reference channel data being registered simultaneously with the working channel. Immobilization of the EBV proteins was performed within the protective-orienting coating - three-dimensional structure of the polysaccharide dextran hydrogel $(2 \mathrm{mg} / \mathrm{ml}$ of Dextran 17000 from Sigma in $0.05 \%$ citrate buffer at $\mathrm{pH}$ 5.0...5.2) deposited onto the gold film surface on glass substrate, and the remaining free sites were blocked with bovine serum albumin molecules $(1 \%$ BSA in citrate buffer at $\mathrm{pH}$ 5.0..5.2) to prevent nonspecific interaction.

It has been shown that the developed SPR method in dual channel version can be used for rapid automated diagnosis of herpeviral disease caused by Epstein-Barr virus in population, on a par with linked immunosorbent assay.

\section{Acknowledgments}

The author would like to thank the employees of D. Zabolotny Institute of Microbiology and Virology of NAS of Ukraine: N.V. Nesterova, S.D. Zagorodnya, G.V. Baranova, A.V. Golovan, and the employees of V. Lashkaryov Institute of Semiconductor Physics of NAS of Ukraine Yu.V. Ushenin and A.V. Samoylov for their assistance in performing this work.

This work was supported by the National Academy of Sciences of Ukraine through the project "Pilot operation and metrological support diagnostic instrument based on surface plasmon resonance for the diagnosis of herpes virus. Design and manufacture of sensing elements, electronic, mechanical and software modules devices based on surface plasmon resonance".

\section{References}

1. A. Brecht, G. Gauglitz, Optical probes and transducers // Biosensors and Bioelectronics, 10, p. 923-936 (1995).

2. D.R. Shankaran, K.V. Gobi, N. Miura, Recent advancement in surface plasmon resonance immunosensors for detection of small molecules of biomedical, food and environmental interest // Sensors and Actuators B, 121(1), p. 158-177 (2007).

3. J. Zhang, L. Zhang, W. Xu, Surface plasmon polaritons: physics and applications // J. Phys. D: Appl. Phys. 45(11), p. 113001 (2007).

4. H. Raether, Surface Plasmon on Smooth and Rough Surfaces and on Gratings. Berlin Heidelberg New York London Paris Tokyo, Springer-Verlag, 1988. 
5. B. Koening, M. Graetzel, A novel immunosensor for herpes viruses // Anal. Chem. 66, p. 341-344 (1994).

6. N. Rojo, G. Ercilla, I. Haro, GB virus C (GBV-C)/ hepatitis $\mathrm{G}$ virus (HGV): Towards the design of synthetic peptides-based biosensors for immunediagnosis of GBV-C/HGV infection // Current Protein and Peptide Sci. 4(4), p. 291-298 (2003).

7. H. Vaisocherova, K. Mrkvova, M. Piliarik, P. Jinoch, M. Steinbachova, J. Homola, Surface plasmon resonance biosensor for direct detection of antibody against Epstein-Barr virus // Biosensors and Bioelectronics, 22(6), p. 1020-1026 (2007).

8. A.K. Junker, Epstein-Barr Virus // Pediatrics in Review, 26, №3, p. 79-84 (2005).

9. S.E. Godstall, J.T. Kirchner, Infectious mononucleosis. Complexities of common syndrome // Postgrad Med. 107(7), p. 175-186 (2000).

10. R. Khanna, S.R. Burrows, Immune regulation in Epstein-Barr virus-associated diseases // Microbiol. Revs. № 9, p. 387-405 (1995).

11. A.M. Yegorov, A.P. Osipov, B.B. Dzantiev, Theory and Practice of Immune-enzyme Analysis. Vysshaia Shkola, Moscow, 1991 (in Russian).

12. G.G.B. Klaus (Ed.). Lymphocytes: A Practical Approach. Oxford; Washington, DC, IRL Press, 1997, p. 261.

13. B. Byrne, E. Stack, N. Gilmartin, R. O'Kennedy, Antibody-based sensors: Principles, problems and potential for detection of pathogen and associated toxins // Sensors, 9, p. 4407-4445 (2009).
14. N.V. Nesterova, L.M. Nosach, S.D. Zagorodnya, O.Y. Povnitsa, P.M. Boltovets, G.V. Baranova, A.V. Golovan, Elaboration of optical immunesensors based on the surface plasmon resonance for detecting specific antibodies and antigens of Epstein-Barr virus and human adenovirus // Microbiology J. 70(6), p. 67-73 (2008).

15. A.A. Kolomenskii, P.D. Gershon, H.A. Schuessler, Sensitivity and detection limit of concentration and adsorption measurements by laser-induced surfaceplasmon resonance // Appl. Opt. 36, p. 6539-6547 (1997).

16. K. Lin, Y. Lu, Z. Luo, P. Wang, H. Ming, Numerical and experimental investigation of temperature effects on the surface plasmon resonance sensor // Chinese Opt. Lett. 7, No. 5, pp. 428-431 (2009).

17. R.B.M. Schasfoort, A.J. Tudos, Handbook of Surface Plasmon Resonance. Cambridge, U.K. Royal Society of Chemistry, 2008, p. 403.

18. N.V. Nesterova, S.D. Zagorodnya, G.V. Baranova, A.V. Golovan, Yu.V. Ushenin, R.V. Khrystosenko, Immunosensor test-system based on the surface plasmon resonance for detecting antibodies against Epstein-Barr virus // Patent on useful model 51125, Ukraine, CI A61K 31/505. Publ. 12.07.2010. Bul. №13.

19. R.V. Khrystosenko, Optimization of the surface plasmon resonance minimum detection algorithm for improvement of method sensitivity // Semiconductor Physics, Quantum Electronics and Optoelectronics, 18(3), p. 279-285 (2015). 